\title{
gpustats: GPU Library for Statistical Computing in Python
}

\author{
Andrew Cron ${ }^{\ddagger *}$, Wes McKinney
}

\begin{abstract}
In this work we discuss gpustats, a new Python library for assisting in "big data" statistical computing applications, particularly Monte Carlobased inference algorithms. The library provides a general code generation / metaprogramming framework for easily implementing discrete and continuous probability density functions and random variable samplers. These functions can be utilized to achieve more than 100x speedup over their CPU equivalents. We demonstrate their use in an Bayesian MCMC application and discuss avenues for future work.
\end{abstract}

Index Terms-GPU, CUDA, OpenCL, Python, statistical inference, statistics, metaprogramming, sampling, Markov Chain Monte Carlo (MCMC), PyMC, big data

\section{Introduction}

Due to the high theoretical computational power and low cost of graphical processing units (GPUs), researchers and scientists in a wide variety of fields have become interested in applying them within their problem domains. A major catalyst for making GPUs widely accessible was the development of the general purpose GPU computing frameworks, [CUDA] and [OpenCL], which enable the user to implement general numerical algorithms in a simple extension of the $\mathrm{C}$ language to run on the GPU. In this paper, we will restrict our technical discussion to the CUDA architecture for NVIDIA cards, while later commenting on CUDA versus OpenCL.

As CUDA and OpenCL provide a C API for GPU programming, significant portions of the development process can be quite low level and require large amounts of boilerplate code. To address this problem, [PyCUDA] and [PyOpenCL] provide a high-level Python interface to the APIs, while also streamlining the process of writing and testing new GPU functions, or kernels. PyCUDA and PyOpenCL compile GPU kernels on the fly and upload them to the card; this eliminates the need to recompile a $\mathrm{C}$ executable with each code iteration. The result is a much more rapid and userfriendly GPU development experience, as the libraries take care of much of the boilerplate code for interacting with the GPU. They also provide seamless integration with [NumPy], which allows GPU functionality to integrate easily within a larger NumPy-based computing application. And, since code is compiled on the fly, it is relatively straightforward to implement metaprogramming

* Corresponding author: ajc40@stat.duke.edu

$\$$ Duke University

Copyright $\odot 2011$ Andrew Cron et al. This is an open-access article distributed under the terms of the Creative Commons Attribution License, which permits unrestricted use, distribution, and reproduction in any medium, provided the original author and source are credited. approaches to dynamically generate customized GPU kernels within a Python program.

In this paper, we discuss some of the challenges of GPU computing and how GPUs can be applied to statistical inference applications. We further show how PyCUDA and PyOpenCL are ideal for implementing certain kinds of statistical computing functions on the GPU.

\section{Development Challenges in GPU Computing}

While a CPU may have 4 or 8 cores, a latest generation GPU may have 256, 512, or even more computational cores. However, the GPU memory architecture is highly specialized to so-called single instruction multiple data (SIMD) problems. This generally limits the usefulness of GPUs to highly parallelizable data processing applications. The developer writes a function, known as a kernel, to process a unit of data. The kernel function is then executed once for each unit or chunk of data.

The GPU has a large single global memory store (typically $512 \mathrm{MB}$ to $4 \mathrm{~GB}$ ) with which data sets can be transferred to and from the CPU memory space. However, each group, or block, of threads are assigned a small piece (typically $16 \mathrm{~K}$ to $64 \mathrm{~K}$ ) of ultra low-latency shared cache memory which is orders of magnitude faster than the global memory. Therefore, the main challenge for the developer, outside of writing the kernel function, is structuring the computation to optimally utilize each thread block's shared memory and minimizing reads from and writes to the global memory. Careful coordination of the threads is required to transfer memory efficiently from global to shared. We omit the low-level details of this process and instead refer the interested reader to the CUDA API guide ([NvidiaGuide]). See Figure 1 for a rough diagram of the computing architecture.

As a larger computation is divided up into a grid of thread blocks, a typical CUDA kernel takes the following structure:

- Coordinate threads within a block to transfer relevant data for block from global to shared memory

- Perform computation using (fast) shared memory

- Coordinate threads to transfer results back into global memory

\section{Computational Challenges in Likelihood-based Statistical In- ference}

In most standard and Bayesian statistical models, a probability distribution (or family of distributions) is assumed for each realization of the data. For example, the errors (residuals) in a linear 
Thread Block

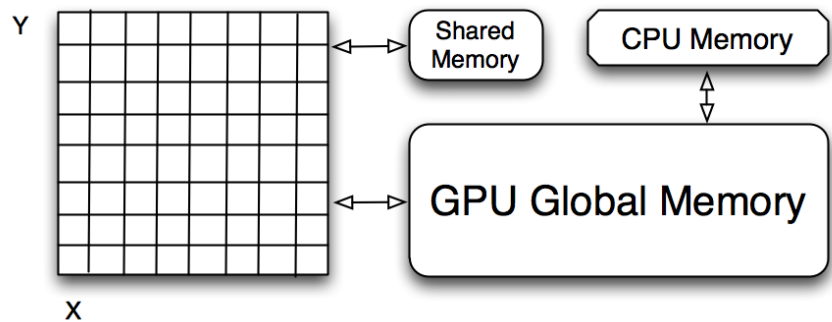

Fig. 1: Mock-up of GPU computing architecture

regression problem are assumed to be normally distributed with mean 0 and some variance $\sigma^{2}$. In a standard statistical inference problem given a set of distributional assumptions, the task is to estimate the parameters of those distributions. Under this setup, we can write down the joint likelihood for the data in mathematical terms

$$
p\left(x_{1}, \ldots, x_{n} \mid \Theta\right)=\Pi_{i=1}^{n} p\left(x_{i} \mid \Theta\right),
$$

where $\Theta$ represents the unknown parameters of the model, and $p\left(x_{i} \mid \Theta\right)$ is the probability density for observation $x_{i}$. This representation assumes that the data are independent and identically distributed. For example, we may wish to estimate the mean $\mu$ and variance $\sigma^{2}$ of a normally distributed population, in which case $\Theta=\left(\mu, \sigma^{2}\right)$ and

$$
p\left(x_{i} \mid \mu, \sigma^{2}\right)=\frac{1}{\sqrt{2 \pi \sigma^{2}}} e^{-\left(x_{i}-\mu\right)^{2} / 2 \sigma^{2}}
$$

In many statistical inference algorithms, the "goodness of fit" of the parameters $\Theta$ is evaluated based on the full data likelihood 1. It is common to use the logged likelihood function as 1 decomposes into a sum of $\log$ densities and this also reduces numerical precision problems.

Many numerical algorithms for fitting these likelihood-based models, especially Monte Carlo-based, involve evaluating the loglikelihood function over thousands of iterations. Thus as the size of the observed data grows, computational expense grows as least linearly in the number of data points. As above, if the data are assumed to be independently generated, the quantity $\log p\left(x_{i} \mid \Theta\right)$ for each observation $x_{i}$ can be evaluated in parallel then summed to compute the full log-likelihood. This becomes a very natural setting for GPUs, and it is quite easy for GPUs to perform even better than large CPU clusters because of the large number of computing cores and very low memory latency. Suchard et al. studied these advantages in the Bayesian mixture model setting and found very promising results (100x speedup) on graphics cards that are now 2 years old ([JCGS]).

Another source of significant computation in statistical applications that we address is that of generating draws from random variables. In many algorithms (e.g. Bayesian Markov Chain Monte Carlo methods), large data sets may require generating thousands or millions of random variables from various probability distributions at each iteration of the algorithm.

\section{Challenges of GPU Computing in Statistical Inference}

As mentioned above, a CUDA or OpenCL programmer must be very mindful of the memory architecture of the GPU. There are multiple memory management issues to address, i.e. in CUDA parlance
- Coalescing transactions between global and shared memory; this is, coordinating groups of typically 16 to 32 threads to copy a contiguous chunk of 64 or 128 bytes in a single transaction

- Avoiding shared memory bank conflicts, i.e. threads competing for read/write access to a shared memory address

To make this more concrete, let's consider the task of implementing the log of the normal probability density function (pdf) 2 . Given a data set with $N$ observations, we wish to evaluate the pdf on each point for a set of parameters, i.e. the mean $\mu$ and variance $\sigma^{2}$. Thus, all that needs to be passed to the GPU is the data set and the parameters. A $\mathrm{C}$ function which can compute the log pdf for a single data point is

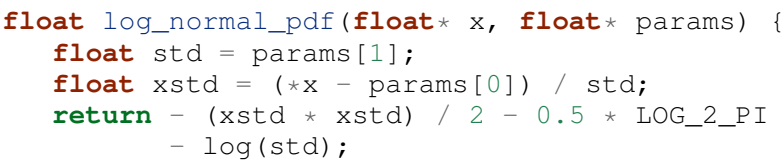

In practice, one would hope that implementing a new probability density such as this would be as simple as writing this 4line function. Unfortunately, to achieve optimal performance, the majority of one's attention must be focused on properly addressing the above data coordination / cache optimization problems. Thus, the full form of a GPU kernel implementing a pdf is typically as follows:

- Coordinate threads to copy (coalesce, if possible) data needed for thread block to shared memory

- Similarly copy parameters needed by thread back to shared memory

- Each thread computes a density result for a single data point, writing results to shared memory

- Copy/coalesce resulting density values back to global memory

Fortunately, the function signature for the vast majority of probability density functions of interest is the same, requiring only data and parameters. While the actual pdf function is very simple, the rest of the code is much more involved. Since the kernels are structurally the same, we would be interested in a way to reuse the code for steps 1,2 , and 4 , which will likely be nearly identical for most of the functions. Were we programming in $\mathrm{C}$, doing so would be quite difficult. But, since we have PyCUDA/PyOpenCL at our disposal, metaprogramming techniques can be utilized to do just that, as we discuss later.

With respect to probability densities, we make a brief distinction between univariate (observations are a single floating point value) and multivariate (vector-valued observations) distributions. In the latter case, the dimension of each observation (the length of each vector) typically must be passed as well. Otherwise, multivariate densities (e.g. multivariate normal) are handled similarly.

In a more general framework, we might wish to evaluate the pdf for multiple parameters at once, e.g. $\left(\mu_{1}, \sigma_{1}^{2}\right), \ldots, . .\left(\mu_{K}, \sigma_{K}^{2}\right)$. In other words, $N * K$ densities need to be computed. A naive but wasteful approach would be to make $K$ round trips to the GPU for each of the $K$ sets of parameters. A better approach is to divide the data / parameter combinations among the GPU grid to maximize data reuse via the shared memory and perform all $N * K$ density computations in a single GPU kernel invocation. This introduces the additional question of how to divide the problem among thread blocks viz. optimally utilizing shared memory. As the available 
GPU resources are device specific, we would wish to dynamically determine the optimal division of labor among thread blocks based on the GPU being used.

Avoiding bank conflicts as mentioned above is a somewhat thorny issue as it depends on the thread block layout and memory access pattern. It turns out in the gpustats framework that bank conflicts can be avoided with multivariate data by ensuring that the data dimension is not a multiple of 16 . Thus, some data sets must be padded with arbitrary data to avoid this problem, while passing the true data dimension to the GPU kernel. If this is not done, bank conflicts will lead to noticeably degraded performance. We are hopeful that such workarounds can be avoided with future versions of GPU memory architecture.

For sampling random variables on the GPU, the process is reasonably similar. Just as with computing the density function, sampling requires the same parameters for each distribution to be passed. Many distributions can be derived by transforming draws from a uniform random variable on the interval $[0,1]$. Thus, for such distributions it makes most sense to precompute uniform draws (either using the CPU or the GPU) and pass these precomputed draws to the GPU kernel. However, there are widelyused distributions, such as the gamma distribution, which are commonly sampled via adaptive rejection sampling. With this algorithm, the number of uniform draws needed to produce a single sample is not known a priori. Thus, such distributions would be very difficult to sample on the GPU.

\section{Metaprogramming: probability density kernels and beyond}

The gpustats Python library leverages the compilation-on-thefly capabilities of PyCUDA and metaprogramming techniques to simplify the process of writing new GPU kernels for computing probability density functions, samplers, and other related statistical computing functionality. As described above in the normal distribution case, one would hope that writing a new density function would amount to writing the simple log_normal_pdf function and having the untidy global-shared cache management problem taken care of by the library. Additionally, we would like to have a mechanism for computing transformed versions of existing kernels. For example, log_normal_pdf could be transformed to the unlogged density by applying the exponent function.

To solve these problems, we have developed a prototype object-oriented code generation framework to make it easy to develop new kernels with minimal effort by the statistical user. We do so by taking advantage of the string templating functionality of Python and the CUDA API's support for inline functions on the GPU. These inline functions are known as device functions, marked by __device__. Since the data transfer / coalescing problem needs to be only solved once for each variety of kernel, we can use templating to generate a custom kernel for each new device function implementing a new probability density. It is then simple to enable element wise transformations of existing device functions, e.g. taking the exp of a logged probability density. In the gpustats framework, the code for implementing the logged and unlogged normal pdf is as follows:

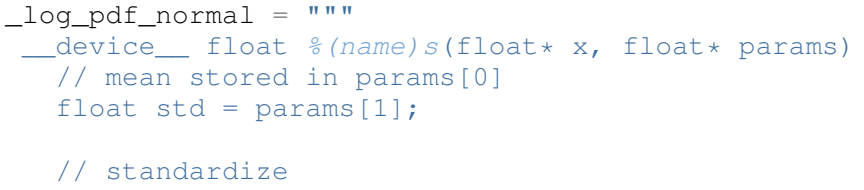

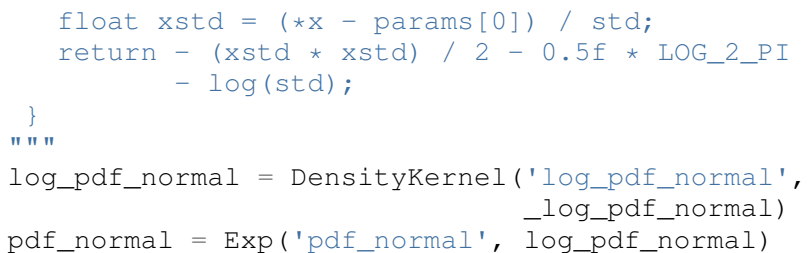

The gpustats code generator will, at import time, generate a CUDA source file to be compiled on the fly by PyCUDA. Note that the $\%$ (name) $\mathrm{s}$ template is there to enable the device function to be given an appropriate (and non-conflicting) name in the generated source code, given that multiple versions of a single device function may exist. For example, the Exp transform generates a one-line device function taking the exp of the logged density function.

\section{Python interface and device-specific optimization}

Further work is needed to interface with the generated PyCUDA SourceModule instance. For example, the data and parameters need to be prepared in ndarray objects in the form that the kernel expects them. Since all of the univariate density functions, for example, have the same function signature, it's relatively straightforward to create a generic function taking care of this often tedious process. Thus, implementing a new density function requires only passing the appropriate function reference to the generic invoker function. Here we show what the function implementing the normal (logged and unlogged) pdf on multiple sets of parameters looks like:

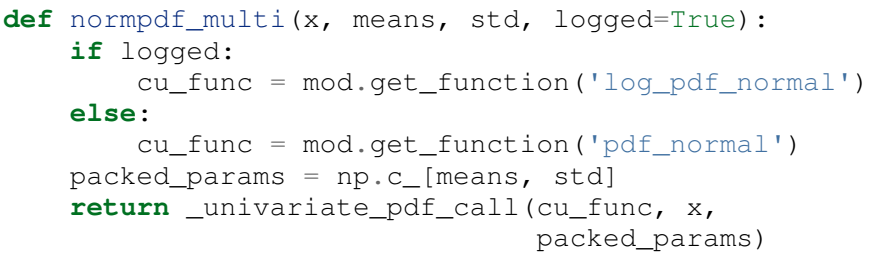

Inside the above _univariate_pdf_call function, the attributes of the GPU device in use are examined to dynamically determine the thread block size and grid layout that will maximize the shared memory utilization. This is definitely an area where much time could be invested to determine a more "optimal" scheme.

\section{Reusing data stored on the GPU}

Since the above algorithms may be run repeatedly on the same data set, leaving a data set stored on the GPU global device memory is a further important optimization. Indeed, the time required to copy a large block of data to the GPU may be quite significant compared with the time required to execute the kernel.

Fortunately, PyCUDA and PyOpenCL have a GPUArray class which mimics its CPU-based NumPy counterpart ndarray, with the data being stored on the GPU. Thus, in functions like the above, the user can pass in a GPUArray to the function which will circumvent any copying of data to the GPU. Similarly, functions like normpdf_multi above can be augmented with an option to return a GPUArray instead of an ndarray. This is useful as in some algorithms the results of a density calculation may be immediately used for sampling random variables on the GPU. Avoiding round trips to the GPU device memory can result in a significant boost in performance, especially with smaller data sets. 


\section{Some basic benchmarks}

We show some benchmarks for the univariate and multivariate normal probability density functions, both with and without using GPUArray to use data already stored on the GPU. These were carried out with a very modest NVIDIA GTS 250 desktop card, which has 128 CUDA cores (latest generation cards have up to 512). The CPU benchmarks were done on a standard Intel Core i7 930 processor. As you will see, the speedups with larger data sets can be quite dramatic. The reported numbers below are the speedup, i.e. the ratio of CPU average runtime divided by GPU average runtime.

Univariate Normal PDF: "Single" indicates that the density values were only computed for a single mean and variance. "Multi" indicates that they were computed for 8 (an arbitrary number) sets of means and variances in one shot. The column header indicates the number of data points.

\begin{tabular}{|c|c|c|c|c|c|}
\hline & & $1 e 3$ & $1 e 4$ & $1 e 5$ & $1 e 6$ \\
\hline Single & & 0.2234 & 1.268 & 7.951 & 23.05 \\
\hline Single & (GPUArray) & 0.2407 & 1.291 & 9.359 & 38.72 \\
\hline Multi & & 1.46 & 7.035 & 26.19 & 43.73 \\
\hline Multi & (GPUArray) & 1.79 & 8.354 & 30.79 & 49.26 \\
\hline
\end{tabular}

Multivariate Normal PDF: For this distribution, we used a streamlined $\mathrm{C}$ implementation of the density function (nearly identical code to the CUDA kernel) for benchmarking purposes so that it's an apples-to-apples comparison. For the data dimension we chose 15 , again arbitrarily. Here we can really see an even greater impact of reusing data on the GPU:

\begin{tabular}{|c|c|c|c|c|c|}
\hline & & $1 e 3$ & $1 e 4$ & 1 e5 & $1 e 6$ \\
\hline Single & & 0.6998 & 4.167 & 12.55 & 14.09 \\
\hline Single & (GPUArray) & 0.8465 & 6.03 & 32.59 & 64.12 \\
\hline Multi & & 3.126 & 18.41 & 60.18 & 63.89 \\
\hline Multi & (GPUArray) & 3.135 & 19.8 & 74.39 & 82 \\
\hline
\end{tabular}

\section{Application: PyMC integration}

Low-hanging fruit for GPU integration in big data applications would be in [PyMC]. This is a library for implementing Bayesian Markov Chain Monte Carlo (MCMC) algorithms. The user describes the generative process for a data set and places prior distributions on the parameters of the generative process. PyMC then uses the well-known Metropolis-Hastings algorithm to approximate samples from the posterior distribution of the parameters given the observed data. A key step in MetropolisHastings is the proposal step in which new parameter values are selected via some proposal distribution, which is typically based on a symmetric random walk but may be more sophisticated. A new proposed value $\theta^{*}$ for $\theta$ is accepted or rejected based on the acceptance ratio

$$
a^{*}=\frac{p\left(\theta^{*}\right) p\left(x \mid \theta^{*}\right) p\left(\theta^{*} \mid \theta\right)}{p(\theta) p(x \mid \theta) p\left(\theta \mid \theta^{*}\right)},
$$

where $p(\theta)$ is the prior density for $\theta, p(x \mid \theta)$ is the likelihood, and $p\left(\theta \mid \theta^{*}\right)$ is the proposal density. Understanding the details of how and why this algorithm works is not important for the scope of this paper. What is important is the fact that the quantity $p(x \mid \theta)$ is recomputed typically thousands of times to compute samples from the model. If the data $x$ is very large, then the majority of the runtime of the MCMC may be spent recomputing the data likelihood for different parameters.

Enabling all of the PyMC distributions to run in GPU mode (so that likelihoods are computed on the GPU) would be very simple as soon as the probability density functions are implemented inside gpustats. Based on the above benchmarks, it is clear that integrating gpustats with PyMC could significantly reduce the overall runtime of many MCMC models on large data sets.

\section{Conclusions and future work}

As gpustats currently uses PyCUDA it can only be used with NVIDIA graphics cards. OpenCL, however, provides a parallel computing framework which can be executed on NVIDIA and ATI cards as well as on CPUs. Thus, it will make sense to enable the gpustats code generator to emit OpenCL code in the near future. As PyOpenCL is developed in lockstep with PyCUDA, altering the Python interface code to use PyOpenCL should not be too onerous. Using OpenCL currently has drawbacks for statistical applications: most significantly the lack of a pseudorandom number generator equivalent in speed and quality to [CURAND]. For simulation-based applications this can make a big impact. We are hopeful that this issue will be resolved in the next year or two.

Another important addition which would be important to some users is to enable multiple GPUs to be run in parallel to extract even better performance. While this would introduce more latency for small datasets and likely be unnecessary, for processing large data sets, the overhead of calling out to 3 GPUs, for example, would likely be much less than the computation time. Ideally code could be seamlessly run on multiple GPUs. Furthermore, the device memory on the GPU can be small. However, most GPUs allow asynchronous memory copying and thread execution, so a streaming approach can be taken on large datasets that can be partitioned. In some cases, the streaming overhead can be virtually eliminated by the asynchronous calls.

Note that gpustats is still in prototype stages, so its API will be highly subject to change. We hope to generate interest in this development direction as it could have an impact in boosting Python's status as a desirable statistical computing environment for big data. An end goal would be to reimplement most of the probability distributions (densities, samplers, etc.) in scipy.stats on the GPU and to fully integrate these where possible throughout PyMC and other related libraries. The meta-programming approach offers a development friendly environment that could also be considered a prototype for a useful GPU programming model in general.

Another interesting avenue, but perhaps of less importance for Python programmers, would be the generation of wrapper interfaces to the generated CUDA or OpenCL source module for other programming languages, such as R. However, without the easy-to-use PyCUDA and PyOpenCL bindings this would likely be a fairly significant undertaking.

\section{Acknowledgements}

We are grateful to Quanli Wang, Jacob Frelinger, Adam Richards, and Cliburn Chan of Duke University for their comments and useful discussions. Research reported here was partially supported by the National Institutes of Health under grant RC1-AI086032. Any opinions, findings and conclusions or recommendations expressed in this work are those of the authors and do not necessarily reflect the views of the NIH.

\section{REFERENCES}

[CUDA] NVIDIA Corporation. CUDA GPU computing framework http://www.nvidia.com/object/cuda_home_new.html 
[OpenCL] Kronos Group. OpenCL parallel programming framework http://www.khronos.org/opencl/

[JCGS] M. Suchard, Q. Wang, C. Chan, J. Frelinger, A. Cron and M. West. Understanding GPU programming for statistical computation: Studies in massively parallel massive mixtures. Journal of Computational and Graphical Statistics 19 (2010): 419-438 http://pubs.amstat.org/doi/abs/10.1198/jcgs.2010.10016

[NvidiaGuide] NVIDIA Corporation. Nvidia CUDA: Programming Guide. (2010), http://developer.download.nvidia.com/compute/cuda/ 3_0/toolkit/docs/NVIDIA_CUDA_ProgrammingGuide.pdf

[CURAND] NVIDIA Corporation. CURAND Random Number Generator http://developer.download.nvidia.com/compute/cuda/3_2/ toolkit/docs/CURAND_Library.pdf

[PyMC] C. Fonnesbeck, A. Patil, D. Huard, PyMC: Markov Chain Monte Carlo for Python, http://code.google.com/p/pymc/

[NumPy] T. Oliphant, http://numpy.scipy.org

[SciPy] E. Jones, T. Oliphant, P. Peterson, http://scipy.org

[PyCUDA] A. Klöckner, http://mathema.tician.de/software/pycuda

[PyOpenCL] A. Klöckner, http://mathema.tician.de/software/pyopencl 